\title{
A NONPRINCIPAL INVARIANT SUBSPACE OF THE HARDY SPACE ON THE TORUS
}

\author{
CHESTER ALAN JACEWICZ ${ }^{1}$
}

\begin{abstract}
Let $H^{2}\left(U^{n}\right)$ be the usual Hardy space (with index 2) of holomorphic functions on $U^{n}$, the unit polydisc in complex $n$-space. A subspace of $H^{2}\left(U^{n}\right)$ is invariant if closed under multiplication by the coordinate functions. To solve a problem left open in a paper of P. R. Ahern and D. N. Clark and a book by W. Rudin the author constructs a closed invariant subspace $M$ of $H^{2}\left(U^{2}\right)$ with (1) an $f$ in $M$ never vanishing on $U^{2}$ and (2) each $g$ in $M$ being contained in a proper closed invariant subspace of $M$. This easily extends to $n \geqq 2$.
\end{abstract}

We answer a question posed in Ahern and Clark's paper Invariant subspaces and analytic continuation in several variables, p. 967 and Rudin's book Function theory in polydiscs, p. 78.

Let $T^{2}$ be the torus with its dual $Z^{2}$ realized as the integral lattice points of the plane under the usual pairing $\left\langle(m, n),\left(e^{i x}, e^{i y}\right)\right\rangle=e^{m i x} e^{n i y}$. For any subset $A$ of $Z^{2}$ let $H^{2}(A)$ be the subspace of $L^{2}\left(T^{2}\right)$ consisting of functions whose Fourier coefficients vanish off $A$. If $A$ is taken to be the set $S=\{(m, n): m \geqq 0, n \geqq 0\}$, then $H^{2}(S)$ is the Hardy space on the torus. Let $U^{2}$ be the set of pairs $(z, w)$ with $z$ and $w$ complex and $|z|<1,|w|<1$. To each function $f$ in $H^{2}(S)$ with Fourier series $\sum_{m \geq 0, n \geq 0} a_{m n} e^{m i x} e^{n i y}$ we associate the function $\sum_{m \geq 0, n \geq 0} a_{m n} z^{m} w^{n}$, analytic on $U^{2}$, which we also denote by $f$.

If $A$ is a subset of $Z^{2}$ and $M$ is a closed linear subspace of $L^{2}\left(T^{2}\right), M$ is $A$-invariant whenever $f$ in $M$ and $c$ in $A$ implies the function $c f$ also lies in $M$. The question we answer is the following: Suppose $M$ is an $S$ invariant subspace of the Hardy space $H^{2}(S)$ and that for each point

Presented to the Society, January 23, 1971 under the title $A$ nonprincipal invariant subspace; received by the editors February 5, 1971.

AMS 1970 subject classifications. Primary 32A30, 46E20, 47D05; Secondary 32A99, 42A92.

Key words and phrases. Hardy space, invariant subspace, outer function, multiple Fourier series, half-plane, semigroup of characters.

${ }^{1}$ This example is contained in the author's Ph.D. thesis, written under the direction of Professor H. Helson at the University of California, Berkeley. The author was partially supported by a National Science Foundation Graduate Fellowship. 
$(z, w)$ in $U^{2}$ there is an $f$ in $M$ (which may vary with $\left.(z, w)\right)$ such that $f(z, w) \neq 0$. Does it follow that there is a single function $h$ such that $M$ is the smallest $S$-invariant subspace containing $h$ ? If such an $h$ exists we will say that $M$ is principal and that $h$ is cyclic for $M$.

The answer is no. Before turning to our counterexample, we include some preparatory remarks. If the subset $A$ of $Z^{2}$ is a semigroup with $A \cup(-A)=Z^{2}$ and $A \cap(-A)=\{(0,0)\}, A$ is called a half-plane. If $h$ lies in $H^{2}(A)$ and the smallest $A$-invariant subspace containing $h$ is precisely $H^{2}(A), h$ is called $A$-outer. For a semigroup $A, H^{2}(A)$ is itself $A$-invariant and any nonzero constant function is $A$-outer. The classical Hardy space is associated with the unit circle, and consists of square integrable functions with Fourier coefficients vanishing off the nonnegative integers. Such a function $\sum_{m \geqq 0} a_{m} e^{m i x}$ also has an extension to an analytic function $\sum_{m \geqq 0} a_{m} z^{m}$ on the open unit disc.

To construct our counterexample we choose $q$ to be a nonconstant, singular inner function in the classical Hardy space of the unit disc [2, p. 67]. This means, first, that $q$ never vanishes inside the unit circle, and, second, that $q$ has modulus one almost everywhere on the unit circle. Now we let $M$ be the $S$-invariant subspace of $H^{2}(S)$ generated by $f$ and $g$, where $f\left(e^{i x}, e^{i y}\right)=q\left(e^{i x}\right)$ and $g\left(e^{i x}, e^{i y}\right)=e^{i y} . M$ meets the requirements for $f(z, w) \neq 0$ for every $(z, w)$ in $U^{2}$, and we will show that $M$ is not principal.

Suppose that $M$ were principal. Let $h$ be cyclic for $M$ and let $M_{1}$ be the $S_{1}$-invariant subspace generated by $h$ under $S_{1}$, the half-plane $\{(m, n): m \geqq$ 0 , and $m=0$ implies that $n \geqq 0\}$. If $q$ is $\sum_{m \geqq 0} a_{m} e^{m i x}$ we see that

$$
\begin{aligned}
a_{0} & =\sum_{m \geqq 0} a_{m} e^{m i x}-\sum_{m \geqq 1} a_{m}\left(e^{m i x} e^{-i y}\right) e^{i y} \\
& =f-\sum_{m \geqq 1} a_{m}\left(e^{m i x} e^{-i y}\right) g
\end{aligned}
$$

lies in $M_{1}$ because $c_{m}=(m,-1)$ lies in $S_{1}$ for $m \geqq 1$, and so $c_{m} g=$ $e^{m i x} e^{-i y} g$ lies in $M_{1}$. But $a_{0}=f(0,0)=q(0) \neq 0$, so that the constant functions lie in $M_{1}$. Thus $h$ is $S_{1}$-outer.

This property of $h$ for any half-plane containing the support of the Fourier transform of $h$ is equivalent to an analytic condition independent of the half-plane. Indeed, if $A$ is a half-plane with $h$ in $H^{2}(A)$, then $h$ is $A$-outer if and only if

$$
\int \log \left|h\left(e^{i x}, e^{i y}\right)\right| d x d y=\log \left|\int h\left(e^{i x}, e^{i y}\right) d x d y\right|>-\infty,
$$

[3, p. 212]. In particular $h$ is also $S_{2}$-outer for $S_{2}=\{(m, n): n \geqq 0$, and $n=0$ implies $m \geqq 0\}$. 
Viewing the situation on the nonnegative integral points of the horizontal axis, one sees that $q$ must be an outer function for the classical Hardy space. Indeed, let $B=\{(m, 0): m \geqq 0\}$ and let $P$ be the orthogonal projection onto $H^{2}(B)$. Now the $S$-invariant subspaces generated by $\{f, g\}$ and $\{h\}$ are the same. Since $S_{2} \supset S$, the $S_{2}$-invariant subspaces generated by $\{f, g\}$ (denoted by $M_{2}(f, g)$ ) and generated by $\{h\}$ (denoted by $\left.M_{2}(h)\right)$ are the same. But $P\left[M_{2}(f, g)\right]$ is the closed linear span of all $e^{m i x} f$, for $m \geqq 0$, while $P\left[M_{2}(h)\right]=P\left[H^{2}\left(S_{2}\right)\right]=H^{2}(B)$. Thus, by the definition of $f$ from $q$, the latter must be outer in the classical Hardy space. But this is known to be false [2, p. 63]. This contradiction means that our assumption that $M$ be principal was incorrect.

\section{BIBLIOGRAPHY}

1. P. R. Ahern and D. N. Clark, Invariant subspaces and analytic continuation in several variables, J. Math. Mech. 19 (1970), 963-969.

2. K. Hoffman, Banach spaces of analytic functions, Prentice-Hall Series in Modern Analysis, Prentice-Hall, Englewood Cliffs, N.J., 1962. MR 24 \#A2844.

3. W. Rudin, Fourier analysis on groups, Interscience Tracts in Pure and Appl. Math., no. 12, Interscience, New York, 1962. MR 27 \#2808.

4. - Function theory in polydiscs, Benjamin, New York, 1969. MR 41 \#501.

Department of Mathematics, Johns Hopkins University, Baltimore, Maryland 21218 